\title{
A SUMMARY OF SASKATCHEWAN CHRISTMAS BIRD COUNTS: 1942-1986
}

WAYNE E. RENAUD, 9 Oakwood Avenue North, Mississauga, Ontario L5G 3L6 GUY J. WAPPLE, \#303-614 9th Street East, Saskatoon, Saskatchewan S7H OM5 and ROBERT D. WAPPLE, Box 1153, Biggar, Saskatchewan. SOK OMO

Recent comprehensive reviews of Christmas Bird Counts in Saskatchewan were published in $1971^{2}$ and 19793; these papers summarized information from 946 counts published during the 1942 to 1976 period. Since then 637 additional counts have been taken. Given the difficulty of keeping track of this volume of information, we felt it was time to compile and present an abbreviated summary of all 45 years of counts (1942 to 1986 ).

Information on frequency, abundance and high counts for the 146 recognized species that were recorded during this period are presented in Table 1.

In compiling this information the same procedures set out in the previous reviews were followed. Where duplicate counts existed with the same name in the same year, we used only one of the two or three counts (that with the highest "count day" species total); observations reported on the other count(s) were treated as "count period" records. Because of this procedure, the actual number of counts used was 1534. The column "Total No. Counts" gives the number of counts on which the species was reported on count day or as an additional species reported only during count period; hence it represents presence or absence. The column "Total No. Count Day individuals" is the sum of all individuals reported on count day only. (Because birds seen during the count period were not included here, the number of counts sometimes exceeds the number of individuals.)
Where only one or two high counts exist for a species, this information is presented for locality and year. Where there are numerous localities with the same high count, the most northerly or southerly record is listed, depending on the normal range of the species. Dates represent the year in which Christmas was recorded. Hence a record for January 1, 1980 would be listed as "79."

For the purposes of this summary we grouped together Common and unidentified redpolls. Most observers assume that unidentified redpolls are Common Redpolls and report them as such.

While this list of species is impressive, most are extremely rare on Christmas Bird Counts in Saskatchewan. In fact four species made up $78 \%$ of the one and one quarter million individuals seen during these 45 years: in decreasing order of abundance these were House Sparrow (31\%), Snow Bunting (28\%), Bohemian Waxwing $(10 \%)$ and Common Redpoll $(9 \%)$. Fiftyeight species have been recorded fewer than 10 times and 27 of these have been represented by a single individual.

When comparing abundance and frequency among various species keep in mind the differences in detectability between many species. For example Northern Flicker and Brown Creeper are recorded with similar frequency; however, one might assume that because flickers are more raucous and visible, counters will actually record a greater proportion of those present in the count area. 
The Christmas Bird Count in Saskatchewan has the potential to evaluate bird populations historically and geographically. While much of any Christmas Bird Count analysis must remain qualitative, because of the methods by which the information has been gathered, we encourage those seeking more detailed information on winter bird distribution in the province to explore new uses for this large volume of raw information.
1 RENAUD, W.E. and G.J. WAPPLE. 1977. A review of Saskatchewan Christmas bird counts: 1942-76 (Part 1). Blue Jay 35(4):224-239.

2 RENAUD, W.E. and G.J. WAPPLE. 1978. Errata and addenda - Saskatchewan Christmas bird counts: A 35-year review (Part 1). Blue Jay 36(2): 121

${ }^{3}$ RENAUD, W.E. and G.J. WAPPLE. 1979. A preliminary study of vegetation zone and winter bird distribution in Saskatchewan. Blue Jay 37(3):202-208.

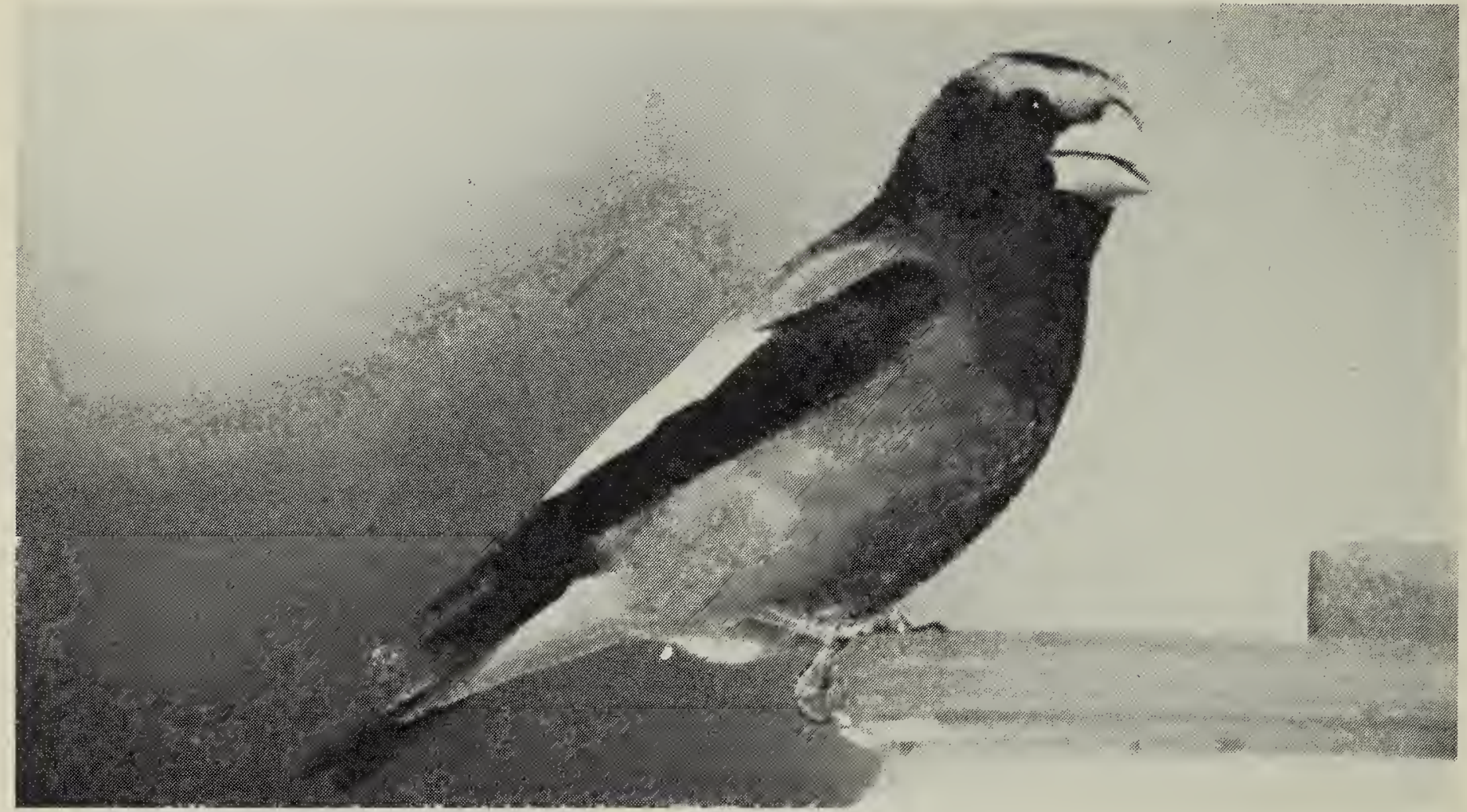

Evening Grosbeak

Lorne Scott

Table 1. SUMMARY OF FREQUENCY, ABUNDANCE AND HIGH COUNTS ON CHRISTMAS BIRD COUNTS IN SASKATCHEWAN, 1942 TO 1986. Species recorded during count period but not on count days are in brackets followed by the notation "CP."

\begin{tabular}{|l|r|c|l|}
\hline Species & $\begin{array}{c}\text { Total } \\
\text { No. } \\
\text { Counts }\end{array}$ & $\begin{array}{c}\text { Total No. } \\
\text { Count Day } \\
\text { Individuals }\end{array}$ & $\begin{array}{l}\text { Highest Count } \\
\text { (Location \& Year) }\end{array}$ \\
\hline Common Loon & 3 & 3 & 1 (Gardiner Dam 82-84) \\
Pied-billed Grebe & 8 & 9 & 2 (Regina 55, 73) \\
Horned Grebe & 5 & 5 & 1 (Regina 77) \\
Red-necked Grebe & 5 & 1 & 1 (Regina 68) \\
\hline Eared Grebe & 3 & 3 & 2 (Regina 62) \\
Western Grebe & & 5 & (Gardiner Dam 79; Regina \\
& 4 & 5 & 2 (Regina 59) \\
\hline American White Pelican & & \\
\hline
\end{tabular}


Table 1. (continued)

\begin{tabular}{|c|c|c|c|}
\hline Species & $\begin{array}{l}\text { Total } \\
\text { No. } \\
\text { Counts }\end{array}$ & $\begin{array}{l}\text { Total No. } \\
\text { Count Day } \\
\text { Individuals } \\
\end{array}$ & $\begin{array}{l}\text { Highest Count } \\
\text { (Location \& Year) }\end{array}$ \\
\hline $\begin{array}{l}\text { Double-crested Cormorant } \\
\text { American Bittern } \\
\text { Great Blue Heron } \\
\text { Tundra Swan }\end{array}$ & $\begin{array}{r}1 \\
1 \\
1 \\
21 \\
\end{array}$ & \begin{tabular}{r|}
1 \\
1 \\
1 \\
80 \\
\end{tabular} & $\begin{array}{l}1 \text { (Squaw Rapids 81) } \\
1 \text { (Yorkton 53) } \\
1 \text { (Ft. Walsh 85) } \\
8 \text { (Regina 65) }\end{array}$ \\
\hline $\begin{array}{l}\text { Trumpeter Swan } \\
\text { Mute Swan } \\
\text { Greater White-fronted Goose }\end{array}$ & $\begin{array}{r}18 \\
1 \\
\end{array}$ & $\begin{array}{r}107 \\
1 \\
\end{array}$ & $\begin{aligned} & 1 \text { (Regina 65; Squaw Rapids } \\
& 85 \text { ) } \\
& 12 \text { (Regina 58) } \\
& 1 \text { (Saskatoon 74) } \\
&\end{aligned}$ \\
\hline $\begin{array}{l}\text { Snow Goose } \\
\text { Ross' Goose } \\
\text { Canada Goose } \\
\end{array}$ & $\begin{array}{r}2 \\
50 \\
\end{array}$ & $\begin{array}{r}2 \\
16486 \\
\end{array}$ & $\begin{aligned} & 1 \text { (Regina } 74,84 ; \text { Saskatoon } \\
&79) \\
& 1(\text { Regina } 62,74) \\
& 1639(\text { Regina } 75) \\
&\end{aligned}$ \\
\hline $\begin{array}{l}\text { Green-winged Teal } \\
\text { American Black Duck } \\
\text { Mallard } \\
\text { Northern Pintail }\end{array}$ & $\begin{array}{r}4 \\
1 \\
208 \\
27 \\
\end{array}$ & $\begin{array}{r}3 \\
1 \\
20 \quad 974 \\
-43 \\
\end{array}$ & $\begin{aligned} 2 & \text { (Saskatoon 74) } \\
1 & \text { (Regina 73) } \\
1225 & (\text { Gardiner Dam 82) } \\
4 & \text { (Saskatoon 79) }\end{aligned}$ \\
\hline $\begin{array}{l}\text { Blue-winged Teal } \\
\text { Northern Shoveler } \\
\text { Gadwall } \\
\text { American Wigeon }\end{array}$ & $\begin{array}{r}6 \\
5 \\
17 \\
13 \\
\end{array}$ & $\begin{array}{r}4 \\
6 \\
18 \\
18 \\
\end{array}$ & $\begin{array}{l}2 \text { (Regina 62) } \\
2 \text { (Regina } 75) \\
2 \text { (Regina 61, 65) } \\
7 \text { (Regina 63) }\end{array}$ \\
\hline $\begin{array}{l}\text { Canvasback } \\
\text { Redhead } \\
\text { Greater Scaup } \\
\text { Lesser Scaup }\end{array}$ & $\begin{array}{r}20 \\
17 \\
1 \\
48 \\
\end{array}$ & $\begin{array}{r}26 \\
35 \\
1 \\
249 \\
\end{array}$ & $\begin{array}{r}4 \text { (Regina 70) } \\
8 \text { (Regina 69) } \\
1 \text { (Regina 74) } \\
15 \text { (Regina -1) } \\
\end{array}$ \\
\hline $\begin{array}{l}\text { Oldsquaw } \\
\text { White-winged Scoter } \\
\text { Common Goldeneye }\end{array}$ & $\begin{array}{r}1 \\
2 \\
128 \\
\end{array}$ & $\begin{array}{r}0 \\
3 \\
3 \quad 620 \\
\end{array}$ & $\begin{array}{l}1 \text { (CP Ft. Qu'Appelle 68)] } \\
2 \text { (Cold River 74); } 1 \text { (Gardiner } \\
\text { Dam 81) } \\
250 \text { (Squaw Rapids 85) }\end{array}$ \\
\hline $\begin{array}{l}\text { Bufflehead } \\
\text { Hooded Merganser } \\
\text { Red-breasted Merganser }\end{array}$ & $\begin{array}{l}5 \\
5 \\
8\end{array}$ & $\begin{array}{r}4 \\
9 \\
11\end{array}$ & $\begin{array}{l}2 \text { (Regina 73) } \\
3 \text { (Estevan 60) } \\
2 \text { (Regina 56; Saskatoon 73; } \\
\text { Gardineı Jarn 86; Squaw }\end{array}$ \\
\hline $\begin{array}{l}\text { Common Merganser } \\
\text { Ruddy Duck } \\
\text { Turkey Vulture }\end{array}$ & $\begin{array}{r}51 \\
15 \\
1 \\
\end{array}$ & $\begin{array}{r}385 \\
42 \\
1\end{array}$ & $\begin{aligned} & \text { Rapids 81) } \\
& 154 \text { (Gardiner Dam 86) } \\
& 7 \text { (Regina 62) } \\
& 1 \text { (Ft. Walsh 84) } \\
&\end{aligned}$ \\
\hline $\begin{array}{l}\text { Bald Eagle } \\
\text { Northern Harrier } \\
\text { Sharp-shinned Hawk } \\
\text { Cooper's Hawk }\end{array}$ & $\begin{array}{r}100 \\
10 \\
11 \\
6\end{array}$ & $\begin{array}{r}266 \\
7 \\
9 \\
2 \\
\end{array}$ & $\begin{array}{l}52 \text { (Squaw Rapids } 81 \text { ) } \\
1 \text { ( } N \text { to Harris, Hawarden) } \\
1 \text { ( } N \text { to Birch Hills) } \\
1 \text { ( } N \text { to Battleford) }\end{array}$ \\
\hline $\begin{array}{l}\text { Northern Goshawk } \\
\text { Swainson's Hawk } \\
\text { Red-tailed Hawk } \\
\text { Rough-legged Hawk }\end{array}$ & $\begin{array}{r}225 \\
1 \\
7 \\
67 \\
\end{array}$ & $\begin{array}{r}248 \\
1 \\
6 \\
56 \\
\end{array}$ & $\begin{aligned} & 16 \text { (Squaw Rapids 80) } \\
& 1 \text { (Webb-Swift Current 78) } \\
& 2 \text { (Squaw Rapids 80) } \\
& 10 \text { (Govenlock 86) }\end{aligned}$ \\
\hline $\begin{array}{l}\text { Golden Eagle } \\
\text { American Kestrel } \\
\text { Merlin } \\
\text { Peregrine Falcon } \\
\end{array}$ & $\begin{array}{r}243 \\
5 \\
184 \\
11 \\
\end{array}$ & $\begin{array}{r}250 \\
2 \\
239 \\
5 \\
\end{array}$ & $\begin{array}{l}12 \text { (Govenlock 86; Ft. Walsh 86) } \\
2 \text { ( } N \text { to Lumsden) } \\
9 \text { (Saskatoon } 85+86) \\
1 \text { ( } N \text { to Squaw Rapids-Carrot }\end{array}$ \\
\hline $\begin{array}{l}\text { Gyrfalcon } \\
\text { Prairie Falcon } \\
\text { Gray Partridge }\end{array}$ & $\begin{array}{r}27 \\
86 \\
841\end{array}$ & $\begin{array}{r}22 \\
58 \\
19937\end{array}$ & $\begin{aligned} & \text { River) } \\
& 3 \text { (Squaw Rapids 82) } \\
& 4 \text { (Govenlock 86) } \\
& 53 \text { " askatoon 70) }\end{aligned}$ \\
\hline
\end{tabular}


Table 1. (continued)

\begin{tabular}{|c|c|c|c|}
\hline Species & $\begin{array}{l}\text { Total } \\
\text { No. } \\
\text { Counts }\end{array}$ & $\begin{array}{l}\text { Total No. } \\
\text { Count Day } \\
\text { Individuals }\end{array}$ & $\begin{array}{l}\text { Highest Count } \\
\text { (Location \& Year) }\end{array}$ \\
\hline $\begin{array}{l}\text { Chukar } \\
\text { Ring-necked Pheasant } \\
\text { Spruce Grouse } \\
\text { Willow Ptarmigan }\end{array}$ & $\begin{array}{r}4 \\
292 \\
32 \\
17 \\
\end{array}$ & \begin{tabular}{r|r|}
44 \\
1885 \\
78 \\
81 \\
\end{tabular} & $\begin{array}{l}30 \text { (Lumsden 58) } \\
55 \text { (Woodrow 59) } \\
29 \text { (Nipawin 48) } \\
32 \text { (Stony Rapids 71) }\end{array}$ \\
\hline $\begin{array}{l}\text { Ruffed Grouse } \\
\text { Sage Grouse } \\
\text { Sharp-tailed Grouse } \\
\text { Wild Turkey }\end{array}$ & $\begin{array}{r}542 \\
25 \\
982 \\
6 \\
\end{array}$ & $\begin{array}{r}1205 \\
248 \\
17512 \\
24 \\
\end{array}$ & $\begin{aligned} & 17 \text { (Battleford 60) } \\
& 106 \text { (Govenlock 79) } \\
& 386 \text { (Harris 72) } \\
& 14 \text { (Ft. Walsh 86) } \\
&\end{aligned}$ \\
\hline $\begin{array}{l}\text { American Coot } \\
\text { Killdeer } \\
\text { Common Snipe } \\
\text { Ring-billed Gull }\end{array}$ & $\begin{array}{r}24 \\
1 \\
7 \\
2 \\
\end{array}$ & $\begin{array}{r}71 \\
1 \\
9 \\
1 \\
\end{array}$ & $\begin{aligned} 11 & \text { (Regina 63) } \\
1 & \text { (Govenlock 82) } \\
3 & \text { (Ft. Walsh 81) } \\
1 & \text { (Saskatoon 58; CP Regina 69) }\end{aligned}$ \\
\hline $\begin{array}{l}\text { [Herring Gull } \\
\text { Iceland Gull } \\
\text { Glaucous Gull } \\
\text { Rock Dove } \\
\end{array}$ & $\begin{array}{r}1 \\
1 \\
7 \\
678 \\
\end{array}$ & $\begin{array}{r}0 \\
1 \\
9 \\
64552 \\
\end{array}$ & $\begin{aligned} & 1 \text { (Cp Regina 56)] } \\
& 1 \text { (Squaw Rapids 79) } \\
& 3 \text { (Gardiner Dam 79) } \\
& 3173 \text { (Saskatoon 86) } \\
&\end{aligned}$ \\
\hline $\begin{array}{l}\text { Mourning Dove } \\
\text { Eastern Screech-owl } \\
\text { Great Horned Owl }\end{array}$ & $\begin{array}{r}13 \\
1 \\
729 \\
\end{array}$ & $\begin{array}{r}10 \\
1 \\
1223 \\
\end{array}$ & $\begin{array}{l}3 \text { (Round Lake 75) } \\
1 \text { (Yorkton 85) [Also } 1 \text { Screech- } \\
\text { owl sp. Ft. Walsh 84] } \\
17 \text { (Raymore 81) }\end{array}$ \\
\hline $\begin{array}{l}\text { Snowy Owl } \\
\text { Northern Hawk-owl }\end{array}$ & $\begin{array}{r}594 \\
29\end{array}$ & $\begin{array}{r}979 \\
23\end{array}$ & $\begin{aligned} & 25 \text { (Regina 79) } \\
& 2 \text { (Masefield 60; Battleford - } \\
& \text { North Battleford 77; Squaw } \\
& \text { Rapids - Sipanok Channel }\end{aligned}$ \\
\hline $\begin{array}{l}\text { Barred Owl } \\
\text { Great Gray Owl }\end{array}$ & $\begin{array}{l}11 \\
21\end{array}$ & $\begin{array}{r}5 \\
19\end{array}$ & $\begin{array}{l}\text { 79; CP Round Lake 85) } \\
1 \text { (S to Regina) } \\
2 \text { (Nipawin 73; Squaw Rapids } \\
\text { 82; Prince Albert National }\end{array}$ \\
\hline $\begin{array}{l}\text { Long-eared Owl } \\
\text { Short-eared Owl } \\
\text { Boreal Owl }\end{array}$ & $\begin{array}{r}13 \\
129 \\
15 \\
\end{array}$ & $\begin{array}{r}9 \\
240 \\
13 \\
\end{array}$ & $\begin{aligned} & \text { Pk. 77) } \\
& 2 \text { (Shaunavon 60) } \\
& 23 \text { (Regina 69) } \\
& 1(\text { S to Indian Head) } \\
&\end{aligned}$ \\
\hline $\begin{array}{l}\text { Northern Saw-whet Owl } \\
\text { Belted Kingfisher } \\
\text { Yellow-bellied Sapsucker } \\
\text { Downy Woodpecker }\end{array}$ & $\begin{array}{r}24 \\
1 \\
1 \\
890 \\
\end{array}$ & $\begin{array}{r}11 \\
1 \\
1 \\
2203 \\
\end{array}$ & $\begin{array}{l}1 \text { ( } \mathrm{N} \text { to Maidstone Bridge) } \\
1 \text { (Ft. Walsh 83) } \\
1 \text { (Saskatoon 78) } \\
25 \text { (Saskatoon 86) } \\
\end{array}$ \\
\hline $\begin{array}{l}\text { Hairy Woodpecker } \\
\text { Three-toed Woodpecker } \\
\text { Black-backed Woodpecker } \\
\text { Northern Flicker }\end{array}$ & $\begin{array}{r}815 \\
75 \\
50 \\
70 \\
\end{array}$ & $\begin{array}{r}1952 \\
117 \\
55 \\
108 \\
\end{array}$ & $\begin{aligned} & 25 \text { (Saskatoon 86) } \\
& 8 \text { (Somme 77) } \\
& 6 \text { (Squaw Rapids 85) } \\
& 9 \text { (Regina 81) } \\
&\end{aligned}$ \\
\hline $\begin{array}{l}\text { Pileated Woodpecker } \\
\text { Horned Lark } \\
\text { Gray Jay }\end{array}$ & $\begin{array}{l}113 \\
422 \\
287\end{array}$ & $\begin{array}{r}87 \\
15 \quad 215 \\
929 \\
\end{array}$ & $\begin{array}{l}3 \text { (Squaw Rapids } 79 \& 86 ; \\
\text { Somme 83) } \\
3043 \text { (Govenlock 85) } \\
67 \text { (Besnard Lake 74) } \\
\end{array}$ \\
\hline $\begin{array}{l}\text { Blue Jay } \\
\text { Black-billed Magpie } \\
\text { American Crow } \\
\text { Common Raven } \\
\end{array}$ & $\begin{array}{r}550 \\
1427 \\
40 \\
414 \\
\end{array}$ & $\begin{array}{rr}2 & 197 \\
36 & 106 \\
& 79 \\
6097 \\
\end{array}$ & $\begin{aligned} & 66 \text { (Togo 77) } \\
& 452 \text { (Saskatoon 86) } \\
& 33 \text { (Prince Albert 57) } \\
& 195 \text { (Besnard Lake 74) } \\
&\end{aligned}$ \\
\hline $\begin{array}{l}\text { Black-capped Chickadee } \\
\text { Mountain Chickadee } \\
\text { Boreal Chickadee } \\
\text { Red-breasted Nuthatch }\end{array}$ & $\begin{array}{r}1209 \\
1 \\
180 \\
144\end{array}$ & $\begin{array}{r}17720 \\
1 \\
835 \\
792\end{array}$ & $\begin{aligned} & 270 \text { (Saskatoon 86) } \\
& 1 \text { (Piapot 66) } \\
& 45 \text { (Waskesiu 82) } \\
& 88 \text { (Ft. Walsh 82) }\end{aligned}$ \\
\hline
\end{tabular}


Table 1. (continued)

\begin{tabular}{|c|c|c|c|}
\hline Species & $\begin{array}{l}\text { Total } \\
\text { No. } \\
\text { Counts }\end{array}$ & $\begin{array}{l}\text { Total No. } \\
\text { Count Day } \\
\text { Individuals }\end{array}$ & $\begin{array}{l}\text { Highest Count } \\
\text { (Location \& Year) }\end{array}$ \\
\hline $\begin{array}{l}\text { White-breasted Nuthatch } \\
\text { Brown Creeper } \\
\text { Golden-crowned Kinglet } \\
\text { Ruby-crowned Kinglet }\end{array}$ & $\begin{array}{r}212 \\
65 \\
74 \\
1 \\
\end{array}$ & $\begin{array}{r}419 \\
92 \\
419 \\
1 \\
\end{array}$ & $\begin{aligned} & 15 \text { (Round Lake 84) } \\
& 7 \text { (Prince Albert 78) } \\
& 45 \text { (Big Gully Creek 74) } \\
& 1 \text { (Biggar 72) } \\
&\end{aligned}$ \\
\hline $\begin{array}{l}\text { Mountain Bluebird } \\
\text { Townsend's Solitaire } \\
\text { American Robin } \\
\text { Varied Thrush }\end{array}$ & $\begin{array}{r}5 \\
15 \\
148 \\
4 \\
\end{array}$ & $\begin{array}{r}4 \\
16 \\
625 \\
2 \\
\end{array}$ & $\begin{aligned} & 2 \text { (Saskatoon 86) } \\
& 3\text { (Regina } 85) \\
& 197 \text { (Saskatoon 78) } \\
& 1 \text { (N to Saskatoon) } \\
&\end{aligned}$ \\
\hline $\begin{array}{l}\text { Northern Mockingbird } \\
\text { Brown Thrasher } \\
\text { Bohemian Waxwing } \\
\text { Cedar Waxwing }\end{array}$ & $\begin{array}{r}1 \\
2 \\
756 \\
81 \\
\end{array}$ & $\begin{array}{rr}1 \\
2 \\
124095 \\
1119 \\
\end{array}$ & $\begin{aligned} & 1 \text { (Regina 67) } \\
& 1 \text { (Saskatoon 60; CP Regina 68) } \\
& 12442 \text { (Saskatoon 75) } \\
& 90 \text { (Saskatoon 75) } \\
&\end{aligned}$ \\
\hline $\begin{array}{l}\text { Northern Shrike } \\
\text { Loggerhead Shrike } \\
\text { European Starling } \\
\text { Yellow-rumped Warbler }\end{array}$ & $\begin{array}{r}282 \\
2 \\
359 \\
2 \\
\end{array}$ & $\begin{array}{r}253 \\
2 \\
6815 \\
2 \\
\end{array}$ & $\begin{aligned} & 6 \text { (Saskatoon 63; Pike Lake 69) } \\
& 1 \text { (Ft. Walsh 81; Regina 82) } \\
& 1000 \text { (Saskatoon 83) } \\
& 1 \text { [Saskatoon } 54 \text { (Myrtle): } \\
&\end{aligned}$ \\
\hline $\begin{array}{l}\text { Northern Cardinal } \\
\text { Rufous-sided Towhee }\end{array}$ & 3 & 3 & $\begin{array}{l}\text { Ft. Walsh } 78 \text { (Aud)] } \\
1 \text { (Craven 60; Saskatoon } 80 \\
\& 81 \text { ) } \\
1 \text { (Moose Jaw 63) }\end{array}$ \\
\hline $\begin{array}{l}\text { American Tree Sparrow } \\
\text { Chipping Sparrow } \\
\text { Field Sparrow] } \\
\text { Vesper Sparrow }\end{array}$ & $\begin{array}{r}72 \\
1 \\
1 \\
1 \\
\end{array}$ & $\begin{array}{r}295 \\
1 \\
0 \\
1 \\
\end{array}$ & $\begin{aligned} & 43 \text { (Ft. Walsh 79) } \\
& 1 \text { (Indian Head 81) } \\
& 1 \text { (CP Saskatoon 85) } \\
& 1 \text { (Saltcoats 52) } \\
&\end{aligned}$ \\
\hline $\begin{array}{l}\text { Fox Sparrow } \\
\text { Song Sparrow } \\
\text { White-throated Sparrow }\end{array}$ & $\begin{array}{r}2 \\
14 \\
14 \\
\end{array}$ & $\begin{array}{r}1 \\
10 \\
16 \\
\end{array}$ & $\begin{array}{l}1 \text { (CP Val Marie 67; Moose } \\
\text { Jaw 85) } \\
1 \text { ( } \mathrm{N} \text { to Pike Lake) } \\
3 \text { (Saskatoon } 81 \text { ) } \\
\end{array}$ \\
\hline $\begin{array}{l}\text { White-crowned Sparrow } \\
\text { Harris' Sparrow } \\
\text { Dark-eyed Junco }\end{array}$ & $\begin{array}{r}3 \\
17 \\
139 \\
\end{array}$ & $\begin{array}{r}2 \\
15 \\
290 \\
\end{array}$ & $\begin{array}{l}1 \text { (Saskatoon 74; Fort Walsh } \\
80 ; \text { CP Broadview 86) } \\
3 \text { (Govenlock 86) } \\
50 \text { (Yorkton 53) }\end{array}$ \\
\hline $\begin{array}{l}\text { McCown's Longspur } \\
\text { Lapland Longspur } \\
\text { Snow Bunting } \\
\text { Red-winged Blackbird }\end{array}$ & $\begin{array}{r}1 \\
30 \\
1 \quad 137 \\
-50 \\
\end{array}$ & $\begin{array}{r}1 \\
574 \\
350 \quad 422 \\
130 \\
\end{array}$ & $\begin{aligned} & 1 \text { (Govenlock 82) } \\
& 200 \text { (Caron 71) } \\
&33935 \text { (Raymore } 82) \\
& 30 \text { (Estevan 62) } \\
&\end{aligned}$ \\
\hline $\begin{array}{l}\text { Western Meadowlark } \\
\text { Rusty Blackbird } \\
\text { Brewer's Blackbird } \\
\text { Common Grackle }\end{array}$ & $\begin{array}{r}20 \\
109 \\
36 \\
20\end{array}$ & $\begin{array}{r}13 \\
428 \\
67 \\
18\end{array}$ & $\begin{array}{c}3 \text { (Skull Creek 71) } \\
83 \text { (Ft. Walsh 86) } \\
23 \text { (Wolseley 63) } \\
3 \text { (Regina 67) }\end{array}$ \\
\hline $\begin{array}{l}\text { Brown-headed Cowbird] } \\
\text { Rosy Finch } \\
\text { Pine Grosheak } \\
\text { Purple Finch }\end{array}$ & $\begin{array}{r}1 \\
27 \\
718 \\
23 \\
\end{array}$ & $\begin{array}{rr} & 0 \\
1139 \\
11996 \\
48 \\
\end{array}$ & $\begin{aligned} & 11 \text { (CP Masefield 59) } \\
& 488 \text { (Ft. Walsh 83) } \\
& 422 \text { (Saskatoon 69) } \\
& 14 \text { (Saskatoon 69) } \\
&\end{aligned}$ \\
\hline $\begin{array}{l}\text { Red Crossbill } \\
\text { White-winged Crossbill } \\
\text { Common Redpoll } \\
\text { Hoary Redpoll }\end{array}$ & $\begin{array}{r}64 \\
110 \\
849 \\
197 \\
\end{array}$ & $\begin{array}{rr}1038 \\
2032 \\
109986 \\
1335 \\
\end{array}$ & $\begin{array}{l}235 \text { (Nipawin 48) } \\
230 \text { (Emma Lake } 75) \\
3651 \text { (Nipawin 52) } \\
62 \text { (Cumberland House 69) } \\
\end{array}$ \\
\hline $\begin{array}{l}\text { Pine Siskin } \\
\text { American Goldfinch } \\
\text { Evening Grosbeak } \\
\text { House Sparrow }\end{array}$ & $\begin{array}{r}31 \\
2 \\
519 \\
1314\end{array}$ & $\begin{array}{r}368 \\
2 \\
12363 \\
388128\end{array}$ & $\begin{aligned} 70 & \text { (Saskatoon 81) } \\
1 & \text { (Saskatoon 79; Regina 81) } \\
515 & \text { (Kamsack 85) } \\
6204 & \text { (Saskatoon 79) }\end{aligned}$ \\
\hline
\end{tabular}

